rev.relac.int.estrateg.segur.7(1):125-148,2012

\title{
LOS NEOCONSERVADORES EN LA ESCENA POLÍTICA INTERNACIONAL: ¿DE DÓNDE PROCEDEN?*
}

\section{RESUMEN}

Este artículo está parcialmente basado en la tesis doctoral El humanismo socialista de Erich Fromm. Cuius regio eius religio, defendida en 2011 en la Universidad de Extremadura por Francisco Javier Ruiz Durán. En él se muestra cómo Estados Unidos y el Reino Unido, en su esfuerzo por contener la influencia soviética sobre la Europa de la segunda postguerra mundial, terminaron creando una ideología política gracias a un gran número de intelectuales, provenientes muchos de ellos de los mismos aparatos de la Internacional Comunista, cuya ala más dura terminó conformando el movimiento neoconservador.

Palabras clave: New York Intellectuals Guerra Fría Congreso por la Libertad Cultural izquierda no comunista neoconservadores.

* Este artículo está parcialmente basado en la tesis doctoral El humanismo socialista de Erich Fromm. Cuius regio eius religio, defendida en 2011 en la Universidad de Extremadura por Francisco Javier Ruiz Durán.

** Doctor en Filosofía. Universidad de Extremadura. Badajoz. España. badajoz@gius.es

*** Doctor en Ciencia Política y de la Administración. Área de Ciencia Política y de la Administración. Departamento de Derecho Público. Facultad de Derecho. Universidad Pablo de Olavide, de Sevilla. Sevilla. España. japer@upo.es 


\title{
NEOCONS AT THE INTERNATIONAL POLITICAL ARENA: WHERE ARE THEY FROM?
}

\begin{abstract}
This article is hinged on F. J. Ruiz Durán's Doctoral dissertation titled Erich Fromm's socialist humanism. Cuius regio eius religio, which was defended in 2011 at the University of Extremadura. This work examines how the US and the UK, in attempting to restrict the soviet influence over Europe after the Second World War, forged a political ideology deriving from a large group of thinkers, most of them linked to the Communist International whose toughest wing shaped the neoconservative movement.
\end{abstract}

Key words: New York Intellectuals, Cold War, Congress for the Cultural Freedom, non-communist left, neoconservatives.

\section{OS NEOCONSERVADORES NO CENÁRIO POLÍTICO \\ INTERNACIONAL: DE ONDE SE ORIGINARAM?}

\section{RESUMO}

Este artigo está parcialmente baseado na tese de doutorado $O$ humanismo socialista de Erich Fromm. Cuius regio eius religio, defendida em 2011 na Universidad de Extremadura por Francisco Javier Ruiz Durán. Ele descreve como os EUA e o Reino Unido, em um esforço por conter a influência soviética sobre a Europa no período pós-guerra, acabaram criando uma ideologia política graças a um grande número de intelectuais, muitos deles provenientes do mesmo aparelho da Internacional Comunista, cuja vertente mais radical derivou no movimento neoconservador.

Palavras-chave: Intelectuais de Nova lorque, Guerra Fria, Congresso pela Liberdade Cultural da esquerda não comunista, neoconservadores 


\section{INTRODUCCIÓN}

El presente artículo tiene como objetivo demostrar el papel de los servicios de inteligencia angloamericanos en el desarrollo político, económico, social y cultural de la Europa de la segunda postguerra mundial para contener al comunismo y favorecer el Estado de Bienestar, y que propició el surgimiento del neoconservadurismo. El artículo muestra cómo Estados Unidos y el Reino Unido, en su esfuerzo de contención de la influencia soviética sobre Europa, terminaron creando una ideología política gracias a un gran número de intelectuales, provenientes muchos de ellos de los mismos aparatos de la Internacional Comunista, cuya ala más dura terminó conformando el movimiento neoconservador.

La metodología se fundamentará en la conjunción de los métodos histórico y comparativo, con el fin de segmentar la complejidad de aquel mundo bipolar para su mejor comprensión. Con el método histórico revelaremos los pilares psicológicos, teológicos, económicos, sociales, políticos e históricos que rodearon al mundo de la Guerra Fría. Una vez mostrados los valores reales de la situación, desbrozaremos el pensamiento y la acción de algunos de los intelectuales más importantes en esta cuestión. Finalmente, con el método comparativo, sopesaremos la viabilidad que tuvo el proyecto de la izquierda no comunista en la vida política internacional con la aparición del movimiento neoconservador.

\section{LA GUERRA FRÍA CULTURAL Y LA POLÍTICA DE LA LIBERTAD}

En 1945 la Unión de Repúblicas Socialistas Soviéticas (URSS) lanzó, con el apoyo de sus servicios secretos, una campaña internacional por la paz con el fin de consolidar sus democracias populares -los países satélites-, como una maniobra defensiva para evitar el conflicto directo con los aliados. Fue la señal para que los servicios de inteligencia americanos y británicos respondieran reclutando, como lo hicieron los soviéticos durante todo el periodo de entreguerras, intelectuales, frecuentemente de ambientes trotskistas, para desarrollar una "ideología que rivalizase con el comunismo", como expresó el primer jefe del Departamento de Investigación de Información (IRD por sus siglas en inglés), Ralph Murray. El reclutamiento les resultó bastante fácil: el propio Stalin les allanó el camino con los procesos de Moscú, el exilio y asesinato de Trotsky y el pacto con Hitler. Sidney Hook, buen exponente de estos marxistas desilusionados, encabezó a los agentes de primer orden, los New York Intellectuals, en el cumplimiento eficaz de las misiones que la Agencia Central de Inteligencia (CIA, por sus siglas en inglés) les confiaban en el marco de la Guerra Fría Cultural'

1. La utilización de la cultura como herramienta de persuasión política para ejercer presión en los asuntos internacionales. Un arma operativa que de forma pacífica busca ganar la batalla por la conciencia de los hombres, entonces, en Europa Occidental. 
A la par, James Burnham ${ }^{2}$ e Irving Kristol ${ }^{3}$, los grandes teóricos de este movimiento, elaboraron el corpus doctrinario de lo que fue, mucho más tarde, el movimiento neoliberal. Entre ellos también debemos apuntar a Daniel Bell ${ }^{4}$ que se unió más adelante.

El Reino Unido, bajo el gobierno de Clement Attlee, creó en febrero de 1948, dentro del Foreing Office, el mencionado IRD para esta nueva estrategia propagandista. Pero antes tuvo que romper los lazos que le unían con la URSS, es decir, tuvo que dar por acabada la relación propagandista que habían llevado juntos durante la Segunda Guerra Mundial, concretamente entre 1942 y 1945.

Los New York Intellectuals tuvieron su bautismo de fuego en la propia Nueva York, en 1949. Como marxistas que eran no tuvieron problemas para integrarse en las filas que asistían a la "conferencia científica y cultural por la paz mundial" auspiciada por Moscú y vigilada por la Kominform, en el Hotel Waldorf, y su trabajo fue un éxito para sus servicios de información. Sidney Hook, uno de los infiltrados, había nacido en Estados Unidos, pero como muchos otros en su época se sintió atraído por la revolución soviética y con una beca posdoctoral pudo viajar a Moscú. En la década de los años treinta tomó partido por la causa de Trotsky e ingresó nada más crearse (1938) en el American Workers Party, como también hizo James Burnham. Pero el trato que la revolución dio a Trotsky lo desilusionó hasta tal punto que abandonó el ideal comunista para fundar una organización antiestalinista en 1939: el Committee for Cultural Freedom, origen del Congreso por la Libertad Cultural (en adelante, también el Congreso). Hook también sentó las bases de la delación y el espionaje por parte de los intelectuales en dos artículos que publicó contra la caza de brujas de McCarthy: "iHerejía sí, conspiración, no!" y "Los peligros de la vigilancia cultural", y fue uno de los grandes teóricos de la Hoover Institution (el Presidente Ronald Reagan le condecoró con la medalla de la libertad). Pronto las filas de los intelectuales se comenzaron a nutrir con egregios nombres: Stephen Spender ${ }^{5}$, Arthur Koestler ${ }^{6}$, André Gide Irving Kristol, Daniel Bell, Melvin Lasky ${ }^{8}$ James Burnham.

2. James Burnham. fue un teórico político estadounidense, que adoptó inicialmente una posición comunista, para luego pasarse a una posición anticomunista. Durante la Guerra Fría escribió regularmente para la revista National Review, de tendencia conservadora. En 1983 recibió la Medalla Presidencial de la Libertad de manos del presidente Ronald Reagan. Sus ideas fueron muy influyentes entre los neoconservadores y los conservadores ortodoxos, facciones de la derecha estadounidense.

3. Irving Kristol publicista de inicios trotskistas que hoy es considerado el fundador del neoconservadurismo estadounidense. Fue editor de la revista Commentary de 1947 a 1952; y cofundador de la revista británica Encounter (financiada en secreto por la CIA); entre otras publicaciones. []. Desde 1988, ha sido miembro del American Enterprise Institute. En julio de 2002 el presidente George W. Bush le otorgó la Medalla Presidencial de la Libertad.

4. Daniel Bell. Sociólogo y profesor emérito de Harvard que estaba entre los Intelectuales de Nueva York, un grupo de escritores de izquierda anti-estalinistas.

5. Stephen Spender. Poeta británico que combatió en la Guerra civil española con las Brigadas Internacionales. Desilusionado fue uno de los fundadores de la revista Horizon y más tarde Encounter. En 1970 fue nombrado profesor de inglés del University College de Londres. 
Gracias a su amistad con este último, Raymond Aron ${ }^{9}$ exportó las tesis de los New York Intellectuals a la vieja Europa y cogió en su regazo al antiguo hombre fuerte del Frente Popular francés, Léon Blum. Junto a Aron estuvieron Michael Crozier -trabajó en Harvard con Henry Kissinger y Richard Neustadt-, Karl Jaspers ${ }^{10}$ y François Mauriac ${ }^{11}$. Su trabajo en el Congreso llegó hasta los partidarios de una Europa Federal como Altiero Spinelli ${ }^{12}$ y Denis de Rougemont ${ }^{13}$, los militantes de la ex Agrupación de los Republicanos (RDR), los intelectuales gaullistas, los intelectuales reclutados y financiados del Centro de Estudios Sociológicos de Francia como Edgar Morin ${ }^{14}$, Georges

6. Arthur Koestler. Fue un intelectual húngaro de origen judío y nacionalizado británico. Miembro de la Komintern y corresponsal de guerra estuvo en España donde fue encarcelado por espionaje y posteriormente liberado por la intervención del Foreing Office. Tras la II Guerra Mundial formaría parte activa del Congreso por la Libertad Cultural financiado por la CIA.

7. André Gide. Escritor francés, que inspiró a escritores como Camus o Sartre, ganador el premio Nobel de Literatura en 1947. Durante la década de 1930, brevemente se convirtió en comunista, pero quedó desilusionado tras una visita a la Unión Soviética. Sus críticas al comunismo le ocasionaron que perdiera varios de sus amigos socialistas, especialmente cuando publicó su libro Regreso de la URSS en 1936. Formo parte activa del Congreso por la Libertad Cultural financiado por la CIA.

8. Melvin Lasky. Fue un periodista, intelectual y editor de The New Leader y del Der Monat. Lasky had founded the latter during the Berlin Blockade in 1948 as an anti-Communist but Leftist, socially progressive journal which became one of the leading Germanophone highbrow socio-political journals for decades, incorporating essays and articles from many Western European and North-American, as well as dissident East Bloc intellectual figureheads, such as George Orwell , Hannah Arendt, Franz Borkenau, Thomas Mann, Arthur Koestler, Raymond Aron , Ignazio Silone, Heinrich Böll , Hans Sahl, Max Frisch, TS Eliot, Saul Bellow, Milovan Djilas, Richard Löwenthal , Peter de Mendelssohn, Hilde Spiel , Hermann Kesten .Lasky había fundado este último durante el bloqueo de Berlín en 1948 con un espíritu anti-comunista, pero de izquierda; socialmente progresista, la revista se convirtió en una de los principales revistas de socio-política de la época. Por otra parte, Lasky fue más conocido por su papel como Jefe de Redacción de la revistaln the Anglosphere, Lasky was best known for his role as Editor-in-Chief of Encounter. Encuentro. He succeeded Irving Kristol, the original editor and founder, in 1958 and helped turn the young magazine into one of the most highly regarded periodicals in Europe. Logró que Irving Kristol, el editor y fundador, en 1958 la colocase entre las más importantes revistas especializadas de Europa. Lasky steered Encounter to represented the point of view of the anti-Communist, anti-Totalitarian Left.En 1967, se reveló que Encuentro y Der Monat fueron dos de las muchas publicaciones que se habían financiado secretamente por la CIA a través del Congreso de la Cultura Libertad (CCF).

9. Raymond Aron. Fue un filósofo, sociólogo y comentarista político francés. Uno de los grandes analistas de la sociedad actual y de la actuación de los intelectuales de izquierda. Trabajó estrechamente con Charles de Gaulle y fue presidente de la Academia de Ciencias Morales y Políticas de Francia.

10. Karl Jaspers. Filósofo alemán que produjo una fuerte influencia en la teología, en la psiquiatría y en la filosofía modernas.

11. François Mauriac. Escritor francés ganador del Premio Nobel de Literatura en 1952. Es conocido por ser uno de los más grandes escritores católicos del siglo XX. Colaboró con el periódico conservador Le Figaro, para pasar más tarde al recién creado L'Expres.

12. Altiero Spinelli. Fundó el Movimiento Federalista Europeo (Movimiento Federalista Europeo). Detenido en 1927, pasó diez años de prisión y seis de confinamiento. Durante su confinamiento en Ventotene, abandonó el comunismo y abrazado el federalismo.

13. Denis de Rougemont. Escritor y filósofo suizo considerado como uno de los grandes pensadores pioneros de la idea de instituir un federalismo europeo.

14. Edgar Morin. Es un filósofo y político francés de origen judeo-español (sefardí). Se vinculó al socialismo gracias al apoyo del Frente Popular en Francia y en España. Tomó parte en la resistencia ante los nazis y se unió al Partido Comunista Francés en 1941. Pero fue expulsado en 1951 debido a un artículo publicado en France Observateur. 
Friedmann ${ }^{15}$, y hombres de la talla de Malraux ${ }^{16}$. Pero hubo muchos más intelectuales europeos que se reciclaron de su pasado en la propaganda comunista de la Komintern, bajo las alas del Congreso: Salvador de Madariaga ${ }^{17}$, Julián Gorkin ${ }^{18}$, Víctor Alba ${ }^{19}$ y George Orwell, entre otros, y también lo hicieron revistas, libros, obras artísticas y musicales, enciclopedias, círculos de lectores, seminarios, becas, intercambios académicos y programas de investigación.

En esta línea los estadounidenses y sus compañeros británicos pronto comprendieron que para controlar a los sindicatos, los partidos de izquierda y los movimientos intelectuales contrarios a la OTAN debían utilizar una "cuña de la misma madera": el socialismo democrático, la izquierda no comunista que podían desarrollar con los marxistas desilusionados que ahora dirigían el Congreso por la Libertad Cultural en el marco de la Guerra Psicológica de la Guerra Fría Cultural. Éste fue el entorno donde dio a luz el proyecto de la izquierda no comunista, la tercera fuerza que nos ha relatado Graham Greene en "El americano impasible" o, si se prefiere, la tercera vía.

Arthur M. Schlesinger ${ }^{20}$ escribió en 1949 "La política de la libertad. El centro vital", en plena transición del liberalismo americano de posguerra, con la doble tarea de redefinir su posición hacia el comunismo y de reconstruir las bases de la filosofía política liberal para dicho fin. Frente a la convicción marxista de la inevitabilidad del triunfo comunista, basada en la teoría de que el capitalismo lleva dentro de sí la semilla de su propia destrucción, Schlesinger esgrimió las herramientas que ha desarrollado el Estado democrático liberal para crear una sociedad heterogénea, cimentada en un capitalismo democrático y regulado: (i) la redistribución de la riqueza, que desmiente la predicción de la miseria progresiva que preveía el marxismo;

15. Georges Friedmann. Sociólogo francés, fue uno de los fundadores de la sociología del trabajo contemporáneo tras la II Guerra Mundial. Durante la guerra, fue un intelectual marxista. Friedmann dedicó la mayoría de su trabajo a estudiar las relaciones entre el hombre y la máquina en las sociedades industriales en la primera mitad del siglo XX.

16. André Malraux. Novelista, aventurero y político francés. Miembro de la Komintern llegó a España al comienzo de la Guerra civil, para ponerse a disposición del Gobierno de la II República. Como muchos intelectuales de su época terminó teniendo un sentimiento anticomunista y formando parte del entramado cultural norteamericano.

17. Salvador de Madariaga. Fue un político e historiador español. Durante la Segunda República Española fue ministro y en el exilio formaría parte de la Revista Cuadernos publicada en Hispanoamérica con fondos de la CIA.

18. Julián Gorkin. Fue periodista, político español y agente de la Komintern. Desilusionado del comunismo participó en la edición de tres libros de denuncia de ex militantes comunistas españoles: La vida y la muerte en la URSS, de Valentín González; Yo fui un ministro de Stalin, de Jesús Hernández y Hombres made in Moscú, de Enrique Castro Delgado. A partir de 1953 participó en el Congreso por la Libertad de la Cultura (CLC), organización de intelectuales con un perfil político anticomunista, y dirigió su publicación para Hispanoamérica, la Revista Cuadernos, hasta febrero de 1963.

19. Víctor Alba. Fue un político, periodista, escritor y profesor universitario español, de orientación comunista, que en su exilio en Hispanoamérica colaboró en la Revista Cuadernos.

20. Schlesinger Arthur Meier Jr. Miembro de la Oficina de Información de Guerra, precursora de la CIA, durante la II Guerra Mundial. From 1943-1945 he served in a spy ring operated by the Office of Strategic Services, a precursor to the $\underline{\mathrm{ClA}}$; In Garry Wills 's 1970 book, "Nixon Agonistes", he mentions Schlesinger's background in the OSS. [ 3 1 Dr.Posteriormente formó parte de la red de inteligencia que diseñaba la Guerra Fría Cultural occidental. 
(ii) la estabilización de las crisis económicas, que desmiente la predicción marxista de la evolución de las crisis hasta el colapso económico del capitalismo; y (iii) el control del ciclo económico y la distribución de ingresos a favor de los más desfavorecidos de la sociedad, que permitirán al nuevo capitalismo manejar mejor las consecuencias, a largo plazo, de la modernización.

'La onda del futuro', ha dicho Walter Lippmann, 'no es la dominación comunista del mundo. La onda del futuro es la reforma social y la revolución social que nos lleve a la meta de la independencia nacional y la igualdad de estatus personales' [...] Todos estamos en deuda con J. K. Galbraith por su demostración de que la sociedad de la abundancia exige una profunda reconsideración de la política económica y social [...] La lección final de la sociedad de la abundancia es seguramente que con la abundancia no basta; que la resolución de los problemas cuantitativos de la vida sólo sirve para resaltar la importancia de la calidad de la forma de vida" (Schlesinger 1972: 14)

Es decir, el liberalismo debe tener en cuenta los aspectos cualitativos de la vida, aún cuando estos aspectos están solo marginalmente dentro del radio de acción del gobierno.

En la URSS de los años treinta la industrialización se realizó a costa de la hambruna y el exterminio. El experimento soviético en su apogeo coincidió con la emergencia del fascismo; el hombre, en realidad, imperfecto, demostró sus más bajos instintos.

La lección, en este caso, es el rechazo incondicional del totalitarismo y la reafirmación íntegra del individuo como la fe fundamental del liberalismo contemporáneo. Y en todo momento el liberalismo debe ser protegido tanto del comunismo como de la reacción.

Esto obligó al liberalismo a buscar en sus viejos principios la renovación de la fe en la democracia, sobre el pilar del vigor moral, desde la cual construir una sociedad donde el hombre sea libre y feliz. Esta búsqueda tuvo que comenzar por entender cómo el hombre occidental del siglo XX se había convertido en un ser inseguro, a la deriva, en tensión.

Los cimientos de su civilización, ideas e instituciones se habían resquebrajado bajo sus pies. Algunos pueblos, precipitadamente, reemplazaron esa inseguridad por unas ilusiones de seguridad que ahora les envolvían en una situación parecida de precariedad. En este siglo de la expansión de la libertad, la democracia y la abundancia se pasó, trágicamente, al totalitarismo, al hombre en masa, a los campos de concentración, a la posibilidad de una guerra atómica y a la elección inevitable entre capitalismo o comunismo.

Los dos sistemas han sido acusados de deshumanizar al hombre y de destruir su libertad individual y política. Pero en aras de la verdad, el cambio general de la forma de vida arranca del 
desarrollo tecnológico inmenso que se ha producido en los últimos siglos, que produjo terribles problemas de reajustes para la civilización.

Es decir, la industrialización, nacida de la liberación del hombre del Renacimiento y la Reforma, que proporcionó nuevas libertades y oportunidades para el desarrollo, terminó despersonalizando las relaciones económicas en detrimento de la libertad del individuo; destruyó la ética personal de la sociedad descentralizada en aras de las nuevas prácticas económicas, impersonalizando los sufrimientos que produce. En esa línea, de forma natural, el monopolio de las corporaciones, al contrario del Estado democrático, que no estaban sometidos al control popular desarrolló una filosofía impersonalizada que llegaría a su cenit con el establecimiento de los Estados totalitarios.

El resultado fue poner armas potentes en manos del orgullo y la codicia del hombre, del sadismo y el masoquismo, del éxtasis del poder y del éxtasis de la sumisión; y todo ello, a su vez, incrementó la sensación de culpabilidad del hombre. El resultado fue la aparición de problemas organizativos para los que el hombre no estaba preparado; y ello, por su parte, a su vez, multiplicó las angustias del hombre. El resultado fue acabar con la vitalidad de las viejas religiones sin producir nada nuevo capaz de controlar el orgullo y el poder; y ello, a su vez, agravó al máximo tanto la culpabilidad como la angustia (Schlesinger 1972: 28)

La tensión de la industrialización provocó la angustia del hombre occidental, y el miedo a la libertad lanzó a la sociedad de la época en los brazos del totalitarismo, que lejos de resolver los problemas de organización, los llevó a su apogeo, aniquilando sistemáticamente las diferencias y rivalidades que hacen posible la libertad en una sociedad organizada de forma menos rígida. Es más, el totalitarismo destruyó la libertad sin proporcionar seguridad.

Por ello, la confrontación bipolar que sufrió la Humanidad fue la lucha por las mentes y los corazones. Su objetivo fue fortalecer y defender la sociedad libre. Los medios fueron, en primer lugar, comprender los motivos del fracaso de la sociedad libre para, en segundo lugar, examinar en profundidad el desafío totalitario; y finalmente, planificar la estrategia y la táctica para desarrollar la contraofensiva democrática.

En este sentido debemos comprender que el corazón de las creencias sentimentales del progresismo lo ocupa su fe en el progreso, fruto de la llustración, así como en el unitarismo, la tolerancia y la perfectibilidad humana para desarrollar un verdadero paraíso sobre la tierra en la línea de Darwin, Spencer y Marx. Pero en la tranquilidad del siglo XIX también hubo quien criticaba el rechazo de la oscuridad humana: Dostoievsky, Kierkegaard, Nietzsche, Sorel, y Freud; y, desgraciadamente, el siglo XX demostró que el progreso traicionó a los progresistas. La historia abandonó a sus devotos y desencadenó el terror. Pero el problema de la sociedad moderna radica precisamente ahí, porque no ha sabido construir una nueva estructura organizativa que 
permita y arrope la autorrealización del ser humano. Esto significa que el hombre no es libre per se, que la libertad trae consigo más frustraciones y aislamiento que satisfacciones o sentimiento de integración.

En este sentido Kierkegaard afirmaba que "la angustia es el vértigo de la libertad", pues el hombre debe hacerse a sí mismo eligiendo o destruyendo su personalidad moral, bajo su absoluta responsabilidad, como indicaba Jean-Paul Sartre.

Pero esta filosofía de la responsabilidad, que llamamos libertad, es una carga insoportable para la mayoría de los hombres, que prefieren huir de la elección, huir de la ansiedad, huir de la libertad. Esa huida es lo que Erich Fromm denominó "el miedo de la libertad". Y fue precisamente ese miedo el que llevó a millones de seres humanos a rendir su individualidad a una autoridad externa y masiva.

Por tanto, el Estado totalitario ha nacido como respuesta específica a este temor que inspira la libertad; es una creación del siglo XX. El dominio del Estado totalitario es ilimitado. Por otro lado, las simpatías democráticas, inicialmente, se inclinan más por el totalitarismo soviético que por el fascismo. La tradición humanitaria inteligible, durante un tiempo, de sus esperanzas e ideales justifica, junto a la magnitud de sus problemas y a los ataques de la reacción, la dureza de sus métodos. Pero la dureza aplicada por Lenin en pro de la inevitabilidad de la historia concedió a la revolución soviética un carácter de consagración sacrificada al bien de la humanidad. Una consagración que llevaba tanto las simientes del absolutismo estalinista como el oprobio que desilusionó a tantos comunistas: la deificación del partido.

\section{LA CAÍDA DEL MITO Y LA CREACIÓN DE LA IZQUIERDA NO COMUNISTA}

Esta innovación leninista de las tesis originales de Marx sobre la deificación del partido comenzó aplacando la existencia, desde los inicios de la Revolución, de los partidos obreros, socialistas, social-revolucionarios y anarquistas, para asentarse en 1921, tras reprimir el alzamiento de los marinos de Kronstadt, que exigían elecciones mediante sufragio secreto, libertad de palabra y prensa para todos los partidos socialistas de izquierda, libertad de reunión para los sindicatos y las organizaciones campesinas, y la liberación de los prisioneros de ideología socialista y anarquista. Lenin reaccionó ante las peticiones con dureza: esas demandas iban encaminadas a destruir el monopolio comunista del poder, y ordenó la supresión sangrienta de los rebeldes. En el posterior congreso del partido se acabó con la democracia interna que existía en su seno. Comenzaron las detenciones, las deportaciones a Siberia y la lucha contra los sindicatos.

Cierto es que Lenin respetó a sus camaradas; sin embargo, sus sucesores ampliaron el radio de acción a cualquier persona o institución que se permitiera un pensamiento independiente. Marx decía que el partido comunista era un partido igual que los demás partidos obreros. Lenin 
creó un partido que no sólo impedía el desarrollo de otros partidos obreros; también lo creó como una fuerza directora de la revolución. Curiosamente, en 1905, junto a Trotsky, el propio Lenin había advertido de los peligros de la deificación del partido: "Quienquiera que intente lograr el socialismo por otro camino que no sea la democracia política llegará inevitablemente al más absurdo y reaccionario de los resultados, tanto política como económicamente" (Schlesinger, 1972: 102).

Finalmente se concretó el inevitable y reaccionario resultado de un Estado Burocrático que acabó con los experimentos de control obrero, los sindicatos, las huelgas y el libre empleo, en cuyo lugar instituyó el ritmo de trabajo, las jornadas largas y los salarios bajos reduciendo al obrero, nominalmente libre, a una servidumbre peor que la de su oponente capitalista. Y ello sin contar la mano de obra esclava de los campos de concentración. Así, no es de extrañar que ese Estado, más que socialista, fuese denominado por muchos como un "colectivismo burocrático".

Y en todo momento el Estado soviético, lejos de ocultarlo, lo proclamada abiertamente en su fraseología religiosa habitual: "Oh, gran Stalin; oh, líder del pueblo, Tú que creaste al hombre, Tú que creaste la tierra, Tú que hiciste jóvenes los siglos, Tú que hiciste las flores en primavera [...]" (Schlesinger 1972: 106). Con la imposición del culto al líder comenzó el último paso para la burocratización del terror en la línea de pensamiento de Hannah Arendt, $<<$ el campo de concentración es la culminación del dominio y la rendición > >; es el culmen del totalitarismo.

En este contexto, la psicosis social inducida por el colectivismo burocrático revivió el shigalovismo de Dostoievsky, el espionaje entre los ciudadanos, la esclavitud, la destrucción de la cultura, la familia y la propiedad; era la reducción de los hombres a una masa con un mínimo común denominador.

Desde el final de la Segunda Guerra Mundial, la URSS comenzó a desarrollar una política expansionista con el fin de manejar mejor las tensiones internas propias de un Estado totalitario. Y en su imperiosa necesidad de ocupar cualquier vacío de poder, principalmente en sus nuevos satélites, los comunistas aplicaron despiadadamente, a su manera, el antiguo principio de las guerras de religión, "cuius regio, eius religio", quien rige el gobierno rige la mente. La URSS no quería que el ejemplo de Tito se expandiese en sus nuevos territorios. A partir de 1917, con la revolución y la personalidad de Lenin, el radicalismo occidental se adentró en una espiral de complejos que propició que la izquierda independiente de todo el mundo aceptase los crímenes que se cometían en la URSS.

No obstante, hubo quienes no tardaron en denunciar el desarrollo totalitario en el experimento soviético, como los socialistas alemanes, Karl Kaustky o la propia Rosa de Luxemburgo.

Las denuncias de Rosa de Luxemburgo tienen algo de profecía. 'La libertad sólo para los partidarios del gobierno, sólo para los miembros del partido -no importa 
cuán grande sea el número de sus afiliados- no es libertad en absoluto... Sin elecciones generales, sin libertad de prensa, de reunión ni de palabra, la vida de toda institución pública languidece, se convierte en una caricatura de sí misma, y aparece la burocracia como único factor disuasorio'. Pero muy pocos la escucharon... Los partidos socialdemócratas, la única alternativa organizada, eran burocráticos a lo blando... Por eso los comunistas pudieron llevar a cabo con brillante éxito su campaña contra la izquierda independiente. Se mostraron unas veces rudos y otras tiernos, dando bandazos asombrosos; pasaron del frente unido desde arriba al frente unido desde abajo; e invocaron continuamente el hecho indiscutible de que la URSS era el único país 'socialista' del mundo (Schlesinger 1972: 168).

Esta táctica del zig-zag escondía el objetivo primordial del comunismo: la desintegración de la izquierda independiente. Lenin la utilizó con los moderados, los kautskistas, los mencheviques e incluso con los comunistas extranjeros, pero siempre con la misma filosofía. Lógicamente, Stalin también apuntó y desarrolló esta lección; el principio fundamental del Partido fue siempre la lucha contra la izquierda democrática, el principal enemigo, más que contra la reacción. Pero, ¿por qué?, porque los socialdemócratas intentaron resolver los problemas del desempleo y la miseria sin esclavizar por ello a las masas. Así, es comprensible cómo todas las colaboraciones entre las distintas fuerzas de la izquierda siempre fracasaran. No había interés en común, sólo el deseo soviético por controlar o destruir a los demás partidos de la izquierda. Pero, sobre todo, por fin, podemos contestar la famosa pregunta que formuló Lenin: "¿Quién para quién?". La burocracia comunista ejercerá el control sobre todos los demás. Karl Kaustky y Rosa de Luxemburgo lo comprendieron.

Y por ello Schlesinger pensaba que la forma de honrarlos era trabajar por el renacimiento de la izquierda democrática, la izquierda no comunista. El proyecto de la izquierda no comunista descansa en la denuncia que realizó Léon Blum sobre los peligros del comunismo y el gaullismo para enfatizar el trabajo de esa "Tercera Fuerza", de los grupos que luchaban por la libertad.

El primer principio de la izquierda democrática fue la afirmación de su fe en la sociedad libre y su absoluto repudio del totalitarismo [...] Sus profetas fueron los escritores que se negaron a tragarse las fantásticas hipocresías implícitas en la defensa del totalitarismo: Silone, con su profunda sensibilidad moral; Gide, con su vibrante sentido de la libertad; Koestler, con su rigurosa e insaciable curiosidad intelectual; Hemingway, que detestaba a la gente que quería arrastrar a los demás a cualquier sitio; Reinhold Niebuhr, con su sentimiento trágico del hombre; George Orwell, con vigoroso buen sentido y su odio a la hipocresía; Edmund Wilson, con su fe en la moral y el gusto estético. Y sus líderes políticos trajeron una nueva virilidad a la vida política, una virilidad hecha de humanidad, y no de crueldad: Franklin D. Roosevelt, con su soberbia jovialidad y su magnífica visión política; Aneurin Bevan, en quien se resume toda la tradición inglesa del inconformismo; 
Walter Rheuter, el dirigente sindical con visión y voluntad. La izquierda no comunista ha traído la poca o mucha esperanza que hay en la vida política actual. Consideremos el caso de Europa. ¿Qué países han logrado un alto grado de estabilidad económica y política? Inglaterra, Dinamarca, Noruega, Suecia, Bélgica, Holanda, Austria. ¿Cuál es la fuerza comunista en esos países? Insignificante, en todos los casos. ¿Qué fuerza tienen los socialistas? En todos esos países el Partido Socialista participa en las tareas de gobierno y controla las federaciones sindicales de la nación. ¿En qué países de Europa Occidental falta una clara estabilidad económica y política? En Francia y en Italia. Y ellos son los únicos de Europa Occidental donde los partidarios comunistas tienen una gran masa de afiliados, donde dominan las centrales sindicales y donde los partidos socialistas están divididos y son débiles (Schlesinger 1972: 182 ss).

La buena salud de la izquierda democrática radica en atenerse, en todo momento, al principio que exige el rechazo incondicional del totalitarismo, y en ser consciente de que siempre existe el peligro de una recaída.

El compromiso moral característico del nuevo radicalismo es su fe en la libertad y el rechazo incondicional del totalitarismo, su compromiso político característico es la creencia en la revisión de la política marxista, una revisión que pone en cuestión particularmente la concentración absoluta de todo el poder político y económico en el aparato del sistema de partido único [...] la opresión sólo puede ser neutralizada por la capacidad de resistencia de las otras clases [... Y Y la resistencia exige ante todo una base independiente desde la que operar. Exige inviolabilidad de lo privado, fondos, tiempo, periódicos, libertad de reunión, verse libre de temores; exige recursos cuyo acceso esté seguro, recursos que permanezcan relativamente inaccesibles a la clase dirigente. En suma la resistencia es posible sólo cuando la base se encuentra claramente separada del Estado [...] Las Constituciones no son por sí solas garantía de libertad política; sólo la edad, el uso y el apoyo de muchos hacen que adquiera fuerza $[. .$.$] Cuando todo el poder se concentra en las altas jerarquías de un solo$ partido, deja de existir una izquierda que sirva de freno a la clase dirigente [...] Las consecuencias del Estado ilimitado son tan fatales para la libertad y la dignidad del individuo que el nuevo radicalismo no tiene más alternativa que actuar con el estado limitado (Schlesinger 1972: 186 ss).

Precisamente en los errores de Marx al analizar el Estado limitado está la causa del fracaso de sus profecías. En vez de convertirse en un comité ejecutivo para dirigir los negocios del mundo ha desarrollado la competencia entre las clases y la redistribución de la riqueza que ha impedido la miseria del proletariado. Y por otro lado, la izquierda democrática se comprometió con el Estado limitado para desarrollar, en el nuevo radicalismo, una nueva política económica que gradualmente nos separe del abismo a favor de la sociedad libre. Es el camino, es la tercera 
fuerza, que respeta el parlamentarismo, las libertades civiles y el establecimiento del ordenamiento jurídico necesario.

Poco a poco, pasada la intoxicación, el nuevo radicalismo vuelve a la filosofía histórica del liberalismo sin olvidar nunca que el viejo orden "cruje" en todo el mundo, que cualquier camino que conduzca a la paz y a la libertad es azaroso, y que un paso en falso puede provocar una guerra atómica o dirigirnos a la senda totalitaria. El nuevo radicalismo pretende luchar por restaurar el sentido del valor de los hechos y de la integridad de la razón. Su éxito inmediato está en la tarea de remodelar la política de Estados Unidos, la única de las dos grandes potencias accesibles a ello.

Con la "vuelta al redil" de los intelectuales que coquetearon con el comunismo se renovó su interés por las fuerzas de la voluntad, desde las teorías de Freud, y así la teología de Barth y Niebuhr reforzó las antiguas verdades del cristianismo para que el liberalismo tuviese unos pilares poderosos que contraponer al comunismo. El resultado fue que Estados Unidos tenía un principio común con la izquierda no comunista: la libertad era tan valiosa que no podía comprometerse con el totalitarismo.

Los estadounidenses recuperaron la tradición del liberalismo clásico que se cimentaba en la responsabilidad política y en una concepción moderadamente pesimista del hombre para su política exterior.

En esta línea, los norteamericanos comprendieron que la única vía realista para enfrentarse al comunismo en Europa era el desarrollo de los partidos socialistas democráticos. Fruto de esta conclusión fue que desde 1947 Eleanor Roosevelt, Leon Henderson, Paul Porter y Wilson Wyatt, miembros del New Deal, se unieron a hombres como Niebuhr, Elmer Davis y Marquis Childs para crear el Americans for Democratic Action (ADA) que desarrollaría la Tercera Fuerza.

Esa Tercera Fuerza fue bautizada en el Departamento de Estado, con la llegada de George C. Marshall, con la denominación críptica NLC (Non-Communist Left). Pero además, la nueva línea maestra del Departamento de Estado de Estados Unidos, la izquierda no comunista, produjo efectos sobre su propio movimiento sindical. Si bien

es cierto que la American Federation of Labor había ayudado generosamente [...] a los partidos socialistas y a las centrales sindicales libres de Europa [...] hombres como Walter Reuther y James B. N. Carey intentaban que el ClO acudiera en ayuda de la democracia europea, pero sus esfuerzos fueron neutralizados durante largo tiempo por el bloque comunista... colocó al $\mathrm{ClO}$ al lado de la AFL, el ADA y el grupo NCL del Departamento de Estado en la tarea de sostener la Tercera Fuerza en Europa. Las elecciones de 1948 fueron la culminación de estas diversas tendencias en la política exterior e interior [...] América se desplazó hacia la izquierda [...] pero 
hacia una izquierda que era rotundamente no comunista [...] la tarea del liberalismo era consagrarse al mantenimiento de las libertades individuales y al control democrático de la vida económica [...] y no tolerar ningún compromiso, ni en la casa ni el exterior, que pusiera en peligro esos dos principios centrales. El liberal americano llegó en 1948 la conclusión de que no se podía confiar en que el hombre, ser imperfecto, utilice el poder absoluto, sea público o privado, con virtud y sabiduría [...] (Schlesinger 1972: 206 ss).

Esta visión fue la que llevó a Theodore Roosevelt a propugnar el gobierno positivo que invocó, más tarde, el sueño del Estado Benefactor. Este Estado debe fomentar las condiciones favorables a las decisiones económicas, sin tomarlas siempre por sí mismo. Porque debe desarrollar para la empresa privada un entorno económicamente favorable para el incremento de la producción, y finalmente dejar que el mercado libre llegue tan lejos como pueda. El socialismo democrático dejó a Marx para trabajar con el nuevo radicalismo propuesto por Keynes, con el fin de crear un Estado que elevase el nivel de actividad económica a partir de una política estable que favoreciese la inversión privada, el consumo, la paz, y que fuese lo suficientemente efectiva como para prevenir el colapso económico. Entendiendo esto se comprenden mejor los motivos por los que el gobierno socialista inglés, que llegó al poder tras Winston Churchill, se abstuviese de invertir los aspectos fundamentales de su política exterior, en aras de su seguridad, al verse obligado a tener en cuenta consideraciones estratégicas y económicas muy concretas, mientras Estados Unidos se centraba en contener la expansión soviética mediante la política de contención para la prevención de la agresión comunista a los países no comunistas y la política de reconstrucción para la eliminación de las condiciones de miseria en los países no comunistas. La primera la conforman la Doctrina Truman ${ }^{21}$ y el Pacto Atlántico ${ }^{22}$, la segunda se desarrolló bajo el Plan Marshall ${ }^{23} ; y$ de las dos vertientes conjuntadas se extrae una lección:

Si las condiciones en el interior de la casa son intolerables, si unas pocas personas viven rodeadas de lujo mientras el resto se arrastra para recoger las migajas de la mesa y duerme en el suelo, alguien facilitará antes o después la entrada a los comunistas. De ahí que al atrancar la puerta debe acompañarse de una limpieza a fondo de la casa, de ahí que nuestra política deba asegurar a los habitantes contra la des-

21. En su Discurso al Congreso, 12 de marzo de 1947, explicó que la política de los Estados Unidos debía de ser apoyar a los pueblos libres que se resistían a ser sometidos por minorías armadas o por presiones exteriores. Y por ello pensaba que los EEUU debían ayudar a los pueblos libres a forjar sus propios destinos en la manera que ellos eligiesen.

22. Recoge los principios acordados por el presidente de Estados Unidos y el primer ministro del Reino Unido, sobre sus esperanzas para el mundo de postguerra, en una reunión celebrada el 14 de agosto de 1941.

23. Un conjunto de medidas y ayudas económicas, desarrolladas por el general Marshall, que en combinación con la Doctrina Truman los norteamericanos implementaron en el mundo libre para reconstruir la economía de mercado y contener el avance de la URSS. 
esperación que engendra el totalitarismo, procurando devolverles la salud política y económica; salud económica significa altos niveles de producción y empleo; salud política significa instituciones libres bajo el imperio de la ley. Esta es la política del Plan Marshall [...] La política de Estados Unidos [...] la fórmula reconstrucción-contención-Plan Marshall más Doctrina Truman- encierra todas las promesas posibles de repeler la amenaza soviética sobre Europa. Con otra década de paz, una federación democrática puede traer una prosperidad duradera y un nuevo vigor a las naciones libres de Europa (Schlesinger 1972: 274).

La tarea era ardua porque la sociedad libre había estado todo el siglo XX a la defensiva, desmoralizada, angustiada, tambaleándose por los golpes del fascismo y el comunismo; y la sociedad libre sobreviviría, en último término, sólo si la democracia generaba en los ciudadanos, aún, una satisfacción emocionalmente viva y rica para neutralizar las angustias que suscita el industrialismo, lo bastante profunda para reunir a sus miembros en la batalla por la libertad y no sólo por la auto-preservación. La libertad debía convertirse en una fe combativa y para crear en la democracia una fe combativa se debía apostar por la fuerza esencial de la democracia: los valores del individuo. La democracia necesitaba un individualismo que se desarrollase de su propia comunidad. El individuo necesitaba tener su propia libertad dentro del contexto social que también necesitaba para desarrollarse completamente en todas sus dimensiones. Sólo al recuperarse la confianza y la solidaridad en el ser humano podría el individuo curar las graves heridas que en su espíritu había gravado a fuego la sociedad industrial. Por tanto, se necesitaba un individualismo que no separase al hombre de la comunidad, una comunidad que arropara al individuo sin sofocarlo.

\section{LA CIA Y LA “OTAN CULTURAL"}

El nuevo radicalismo, que saca su fuerza de una concepción realista del hombre, toma los problemas como vienen, y los ataca pensando que cuanto mayor sea el avance de los valores humanos, más seguras resultarán la libertad y la realización del individuo. Cree en el ataque, y a él se lanza con apasionada intensidad. Desde los años cincuenta la CIA desarrolló las ciencias de la comunicación como elemento primordial dentro de su proyecto de Guerra Psicológica, como un instrumento para recopilar información sobre el enemigo comunista, elaborar propaganda favorable al atlantismo (Organización del Tratado del Atlántico Norte, OTAN), controlar los movimientos de liberación, y desestabilizar a todos aquellos regímenes prosoviéticos que impedían el control estadounidense de la zona. Fue el efecto principal de la estrategia de contención que elaboró el Presidente Truman: propaganda, sabotaje, guerra económica, acciones preventivas, subversión, ayuda paramilitar, asesinatos y demás actos de operaciones especiales como "santo y seña" de la Oficina de Proyectos Especiales u Oficina de Coordinación de Políticas (OPC) -dirigida por antiguos miembros de la Oficina de Servicios Estratégicos (OSS)-, que dependía de la CIA, la cual había creado el propio Truman en 1946 para sustituir a la Oficina de 
Información de Guerra (OWI) y que mandaba Wisner con la aprobación del mismo George Kennan.

En esta guerra surgieron proyectos como el Proyecto Troy, que se orientaron para introducir la "Verdad" tras la cortina de hierro comunista a través de la radio, como fue el caso de La Voz de América, o como el Proyecto Camelot, para facilitar las intervenciones militares y prevenir el riesgo de revolución en el Tercer Mundo como ocurrió en Yemen, el Congo, Chile o Cuba. Por otra parte, los datos científicos se reunirían enrolando universitarios y financiando centros como el Bureau of Applied Social Research (BASR) de la Universidad de Columbia, dirigido por Paul Lazarsfeld y que la CIA financiaba sin tapadera para perfeccionar sus interrogatorios -en especial cuando estalló la Guerra de Corea-, el Institute for Internacional Social Research, de Hadley Cantril, y el Instituto Tecnológico de Massachussets, que financiaba la empresa Ford (en realidad, también la CIA).

Las colaboraciones de estos grupos continuaron en la Public Opinión Quarterly, revista que en 1937 había creado para la OWI De Witt Poole, de Princeton -posteriormente presidente del Comité Nacional por una Europa Libre-, y cuyo consejo de administración estaba compuesto por Paul Lazarsfeld, Hadley Cantril, Rensis Likert y el propio De Witt Poole. En este ámbito se insertaron las tesis de Wilbur Schramm y de Leo Strauss $(2004,2007)$ sobre el mundo de los "buenos" y los "malos", tesis que pretenderán implicar en dicho mundo a los intelectuales que todavía eran neutrales con la divisa de actuar por la paz, la seguridad y la distensión de las relaciones internacionales. Es el neutralismo fomentado por los New York Intellectuals y al que se unieron hombres como Leo Lagrange, becado por la Fundación Rockfeller, o Leo Lowenthal, que se convirtió en uno de los principales colaboradores de la revista.

Raymond Aron, principal abogado del atlantismo en Europa, amigo de Friedrich von Hayek proveniente de una familia judía alsaciana y con inquietudes socialistas y pacifistas-, aceleró con su entrada el éxito ideológico del Congreso por la Libertad Cultural, creando un pensamiento maduro que le llevó a ser consejero del propio Kissinger y de George Kennan. Durante la Segunda Guerra Mundial fue acogido en el Centro de Documentación Social de Francia -financiado por la Fundación Rockefeller-, en el que colaboró con Robert Marjolin, y, tras la desmovilización general, se encaminó a Londres, donde, gracias a la ayuda de Marjolin, entró en el círculo de trabajo de Jean Monnet y fue captado por André Labarthe -hombre de confianza del General de Gaulle- para trabajar en la nueva revista La France Libre. En ella desarrolló las primeras tesis de la intelectualidad anticomunista; su obra "El opio de los intelectuales" fue un llamamiento para que los intelectuales rompieran con el socialismo y se sumasen a la izquierda no comunista de los New York Intellectuals; y se sumergió profundamente en el Reform Club, bastión de los liberales financiados por la Rockefeller: Lionel Robbins, von Hayek, Karl Mannheim y Mises.

Para contrarrestar el Movimiento por la Paz desarrollado por el comunismo como un frente ofensivo, iniciado en Wroclaw (1948) -donde los poemas de Hikmet y Neruda calaron hondo 
en la juventud polaca- y continuado en París (1949) -cuya invitación venía engalanada con una paloma de Picasso-, Estados Unidos y el Reino Unido vieron necesaria la creación de la arquitectura del Congreso por la Libertad Cultural (1950). Aquel año la ciudad de Berlín celebró la primera manifestación del Congreso con tres objetivos: obtener información fiable sobre el bloque soviético, contrarrestar las tendencias neutralistas de Occidente y establecer lazos con los intelectuales residentes en el telón de acero. Con estas líneas de acción se desarrolló una profunda penetración intelectual también en América Latina con la creación de la Revista Cuadernos, bajo la tutela de Gorkin -antiguo líder del Partido Obrero de Unificación Marxista (POUM) en España-, que aglutinó con una rapidez sorprendente a todos los elementos democráticos contra el estalinismo (Glondys 2007). Bien es cierto que el anticomunismo que se expandía en el continente -en 1954 trece de las veinte repúblicas latinoamericanas estaban dirigidas por un régimen militar- seguía la línea argumental del discurso donde John Foster Dulles, con la llegada de Eisenhower al poder, aclaraba que la eliminación de la injerencia comunista en la zona era su principal misión y que desde su óptica siempre sería preferible un régimen fuerte que uno - por progresista que fuese- que permitiese la entrada del comunismo. En honor a la verdad hay que señalar que los miembros de Cuadernos nunca se cansaron de advertir a Washington de que, de no estabilizar la economía, invertir en el desarrollo industrial y apoyar las libertades, se abrirían realmente las puertas al comunismo en América Latina, como en todo el mundo.

La importancia estratégica de América Latina generó otras acciones encubiertas, como la implantación del sindicato American Federation of Labor and Congress of Industrial Organizations (AFL-CIO) -creado por la ClA y el Pentágono-, y que, entre otras acciones, resultó decisivo en la desestabilización de Chile, Guyana y Brasil, y que manipuló hábilmente a todas las fuerzas de izquierda del continente orientándolas contra el comunismo. En 1962 el Presidente Kennedy creó la Agencia de los Estados Unidos para el desarrollo y con ello se reorganizó la AFL-CIO para que desarrollara su trabajo a través de tres organismos distintos que controlasen diferentes zonas geográficas. Otra faceta de esta influencia fue la creación de redes stay-behind de espionaje en Europa. Ya en 1952 saltó la noticia de la existencia en Alemania de un grupo ultraderechista -Bundesdeutscherjungend- que, con el respaldo de los servicios secretos de la propia Alianza Atlántica, estaba preparado para asesinar a los principales líderes de la izquierda si la URSS invadía Europa. La OTAN quería asegurarse de que la URSS no tuviera la oportunidad de formar gobiernos títeres. Era uno de sus planes dentro de los acuerdos secretos del comité -clandestino- de Unión Occidental, que estaba dentro del programa Gladio de la CIA (Ganser 2010), ${ }^{24}$ como reconoció el General Oswald Le Winter, y que fue firmado por Robert Schuman cuando

24. La OTAN, durante la Guerra Fría, creó un Ejército Secreto anticomunista para contrarrestar una hipotética invasión de la URSS sobre la Europa de postguerra. Más tarde, mientras esa invasión no se producía, el poderío de los Partidos Comunistas en Francia e Italia, principalmente, y el terrorismo internacional, avalado por la URSS, atemorizaron al bando occidental hasta el punto que la OTAN utilizó ese ejército para otros fines (Ganser 2010). (se añade referencia bibliográfica según normas APA) 
era el presidente del Consejo. En Estados Unidos todo estaba integrado en la OPC, dirigida por Frank G. Wisner, que instaló el Centro de Guerra Psicológica en Fort Bragg -base de las Boinas Verdes-, donde también estuvo la Escuela de las Américas. A la par de estas redes, el stay-behind creó también la Logia de Propaganda Due (P2), que enrolaba dentro del Gran Oriente, de Italia, a los miembros de la guerra psicológica y los de operaciones especiales que llegó a extenderse entre miles de políticos, militares, financieros, miembros de la Iglesia y artistas. Todos ellos bajo la dirección de Licio Gelli -antiguo oficial fascista, enlace con el X2 de la OSS y miembro de la organización fascista Decima Mas a las órdenes del príncipe Valerio Borghese-, que llegó a ser el enlace principal con el dispositivo atlántico (asistió a la investidura de los Presidentes Carter, Reagan y Bush padre). Esta red fue creada por Allen Dulles para establecer una alianza contra el comunismo. En ella fue primordial la recuperación de los agentes nazis -como el General Reinhard Gehlen-, muchos de los cuales fueron enviados a América Latina con la ayuda de la Santa Sede bajo la tutela de Monseñor Giovanni Battista Montini -luego conocido como Pablo IV-. En Francia lo realizó el padre Franz Stock dentro del Internado de Coudray-Morancez.

Es más, en "OSS y la Escuela de Frankfurt: La vida cultural dañada de los extranjeros", un trabajo de la profesora de Sociología de la Universidad de Nueva York Susan Cavin (2004), se ha afirmado que la relación entre la Sociología, la Psicología y el espionaje representó una de las conductas triangulares más fascinantes de la historia intelectual estadounidense. En un periodo de sólo cuatro años (1941-1945) la OSS y la OWI aprovecharon la creciente caída de las academias europeas para facilitar la huida de sus mejores representantes hacia las universidades estadounidenses.

En julio de 1941 el General Wild Bill Donovan, desde la Coordinadora de Información (COI), que se convirtió en la OSS en 1942 y de la que a continuación surgió la OWI, comenzó a desarrollar sus actividades de tal manera que cuando la Segunda Guerra Mundial finalizó y la OSS se disolvió, su famosa Rama de Análisis e Investigación había crecido hasta los 900 académicos. Como es lógico, luego se trasladaron a la CIA.

En 1976, Ray S. argumentó sobre lo esenciales que resultaron los estudios de Herbert Marcuse como sociólogo de la OSS en la R\&A, sección alemana, en la llamada Guerra Secreta, así como los de otros investigadores en muchas de las ramas de las Ciencias Sociales. En 1989, Barry Katz reveló que la Sección Europea de Investigación y Análisis de la OSS contrató a miembros de la Escuela de Frankfurt como Marcuse, Franz Neumann, Otto Kirchheimer y Felix Gilbert, junto con otros sociólogos como Morris Janowitz, Edward Shils, Barrintong Moore, y el economista Paul Sweezy. En 1996 Heideking Match lo confirmó y destacó la labor de Max Horkheimer en sección alemana. En esta línea, Claus Dieter ha afirmado que muchos de los grandes teóricos sociales del siglo XX fueron contratados, durante la Segunda Guerra Mundial, por los servicios de inteligencia. En Estados Unidos la OSS y la OWI, principalmente, acogieron a cerca de 1.200 intelectuales que huyeron de Alemania después de 1933 provocando el despido de casi la mitad de sus profesores, principalmente en las áreas de ciencias 
sociales, y haciéndoles a muchos trabajar como consultores para completar sus ingresos fuera de la Universidad. Así se confeccionó para la OSS y la OWI una asesoría que analizó el fenómeno del nazismo bajo dos perspectivas: una dirigida por Neumann y otra por Horkheimer, para analizar las consecuencias psicosociales de la obediencia y sus fuentes de violencia. $Y$ con la llegada de Horkheimer es cuando se le dio prioridad al estudio empírico de la mentalidad de los trabajadores de la República de Weimar que había realizado la Escuela de Frankfurt en 1930 para desvelar las causas por las que el proletariado alemán se inclinaba a la derecha, estudio que se había ocultado para no desvelar la inminente victoria del nazismo y que Erich Fromm (en realidad el primer director del estudio) ya en Estados Unidos desempolvó para analizar la situación del campesino mejicano.

Por otra parte, las tres vertientes de esta relación triangular fueron:

a. La primera, obras que indican los principios fundamentales de orientación para el desarrollo de unos principios mínimos de cohesión doctrinal, entre las que encontramos "La revolución de los directores" (Burnham); "El cero y el infinito" y "El ocaso de un ídolo" (Koestler); "La política de la libertad. El centro vital" (Schlesinger); y "1984" (Orwell).

b. La segunda, obras compiladas mediante las colaboraciones de diversos autores sobre una materia concreta, entre las que nos encontramos "El Humanismo socialista" (Fromm), sobre el humanismo de Marx; "El mundo libre en la guerra fría" (Raymond Aron), una comparativa entre la libertad y el totalitarismo donde se pide a Occidente que recupere sus valores morales y espirituales para dotar de nobleza a la lucha contra el comunismo y en el que participaron Carlo Antoni, el propio Aron, Hans Barth, Ernst Bieri, Hans Kohn, Salvador de Madariaga, Peter Meyer, Arthur Montgomery, Wilhelm Röpke, Alexander Rüstow, Paul Wacker y David McCord Wright; "Conversaciones sobre la nueva cultura", que recoge la exitosa serie de entrevistas de $L^{\prime} E x p r e s s$ donde se reflexiona sobre el destino, el futuro y la condición del hombre y en la que participaron Roland Barthes, Fernand Braudel, Georges Friedmann, Friedrich Hacker, François Jacob, Bertrand de Jouvenel, Arthur Koestler, Claude Lévi-Strauss, André Lichnerowicz, Konrad Lorenz, Marshall McLuhan, Herbert Marcuse, André Martinet, Jean Piaget, Georg Picht, Alfred Sauvy, Pierre Thuillier y Alan Watts; y "Ensayos sobre el Apocalipsis", donde continúan las reflexiones sobre la nueva cultura iniciadas en "Conversaciones sobre la nueva cultura" y donde colaboraron Erich Fromm, Arthur Mendel, Ronald Laing, Daniel Cohn-Bendit (Dany el Rojo) y Gabriel Cohn-Bendit, Kenneth Boulding, Philip Slater, Richard Farson, Herbert Marcuse, Norman Brown, Juan Mascaró y Gary Zinder.

c. Y la tercera, obras como "El fin de las ideologías", donde Daniel Bell ha estructurado todo el trabajo intelectual del Congreso por la Libertad Cultural, como las tácticas para penetrar en el monolito soviético: el fomento del faccionalismo en Polonia, Berlín y Hungría; el papel de la socialdemocracia; el estudio del joven Marx -donde se pone como ejemplo a 
seguir al padre Jean-Yves Calvez-; el redescubrimiento de la alienación en la línea de George Lukács; la anonimidad del hombre en la sociedad industrial, como hizo Simmel; o la colisión entre la creatividad humana y las instituciones sociales, como ratificó Freud. Todo ello para definir a una URSS que había construido un marxismo como un simple análisis científico, desprendido de la moral social del propio Marx, para favorecer que su análisis económico se apartase del futuro existencial del hombre que en los acentos de una escatología secular habían guiado al propio Marx para entregarla a una monstruosa burocracia estatal que negaba cualquier acceso a la verdadera ética de la responsabilidad que la "OTAN cultural" deseaba para la nueva izquierda.

En una entrevista Berlin se refirió al humanismo comentando que "[e]s algo que necesitamos hoy más que nunca. Porque sólo a través de la cultura humanista llegamos a ver cómo el fanatismo nos lleva a consecuencias terribles. Sólo en las culturas de este tipo la gente es consciente de que los valores son irreconciliables a veces, y que hay que negociarlos" (García Guitián 2001: 75), y de ahí su defensa de los valores humanistas como un auténtico pilar maestro hacia un orden internacional que represente un mundo moral común. Por ello, Berlin, en una expresión de sinceridad, afirmó que el contenido político de su ensayo "Dos conceptos de libertad" sobre el liberalismo- había estado supeditado al contexto histórico de la Guerra Fría:

[F]ue este fenómeno el que justificó la elección del tema e influyó enormemente en como habría de desarrollarlo. La libertad aparece así, por un lado, como uno de los elementos claves del problema central de la política, el de la coacción y la obediencia, al que los dos sistemas ideológicos hegemónicos en ese periodo daban soluciones, no sólo opuestas, sino antagónicas. De ahí [...] habían originado el gran conflicto ideológico de dicha época [...] El objetivo final que asoma detrás de su planteamiento es mostrar las manipulaciones que los regímenes totalitarios realizaban con el concepto de libertad política (García Guitián 2001: 139).

\section{LA LLEGADA AL PODER DE LOS NEOCONSERVADORES}

En la actualidad, este punto de vista sobre el papel de los intelectuales en la creación del Congreso por la Libertad Cultural está saliendo a la luz paulatinamente. Lo demuestran las referencias a Milan Kundera en El País (Elola, 2008, Octubre 26) y La Razón (2008, Octubre 14) y al final del POUM en La Razón (Pardeiro, 2008, Octubre 6), así como las obras de Tony Judt ("Pasado Imperfecto", 2007; y "Sobre el olvidado siglo XX", 2008), Tim Weiner ("Legado de cenizas. La historia de la CIA", 2008) y Michel Winock ("El siglo de los intelectuales", 2010). Pero tampoco debemos olvidar, por su importancia histórica y por los acontecimientos actuales en el plano internacional, que la disensión interna en el Congreso por la Libertad Cultural entre "duros" y "blandos" fue el germen de un nuevo movimiento político que desde el comunismo fue evolucionando hasta integrarse en el ala izquierdista del Partido Demócrata estadounidense 
-bajo la tutela de uno de los Cowboys de Park Avenue, el mencionado Jackson-, para finalmente cerrar el círculo e integrarse en el Partido Republicano.

Los "duros" del Congreso -James Burnham, Sidney Hook, Arthur Koestler, Irving Kristol, Daniel Bell-, empujados por la llegada a la presidencia del Comité Nacional por una Europa Libre del senador Jackson -el cual integró hábilmente en el Comité a Eisenhower, quien no olvidó el favor-, se sumieron en una actividad febril con la llegada del propio Eisenhower a la Casa Blanca en 1953, porque ello les permitió fomentar los mecanismos del Congreso: New Leader, Sol Levitas, Fortune, el Comité por una Europa Libre o Radio Europa Libre. Pero las diferencias llegaron a tal punto que se apartó a Koestler del Congreso: así nacería la escisión del Comité Americano por la Libertad Cultural, donde recalaron, junto a Koestler, Sidney Hook, Irving Kristol o Sol Stein. Pero el nuevo Comité -a diferencia del Congreso- no tardó en dividirse también a causa de esta polarización de las posiciones. Los conservadores fueron Diana Trilling y el Kibbutz del Upper West Side integrado por James Burnham, Arnold Beichmann, Meter Viereck, Clement Greenberg y Elliot Cohen; y los moderados fueron Arthur Schlesinger, Reinhold Nieburhr, James T. Farell, Richard Rovere Philip Rahv y Norman Thomas.

El Comité era el cuartel general del antiestalinismo que dirigía publicaciones como Commentary, New Leader, Partisan Review y, por supuesto, Encounter. Pero los "duros" volvieron a "romper la cuerda", esta vez por no condenar a McCarthy, y Burham encabezó la salida del ala conservadora del Comité. Esta ala es la que Mary McCarthy -una de las que solicitó al Comité la condena de McCarthy- describió como una amalgama de izquierdistas, anarquistas, nihilistas y oportunistas que se hacían llamar conservadores y con la que se debía acabar si no era demasiado tarde. Pero la propia McCarthy sabía que era tarde: Orwell había entregado antes de morir su cuaderno azul al IRD con la revisión de Koestler y Rees (cosa que ella sabía), el Comité había descubierto a Dios, y Eisenhower incluyó por ello en el juramento de fidelidad la leyenda "Una nación bajo el poder de Dios" y desarrolló la espiritualidad de su "In God We Trust" en unos billetes que fueron los pilares de la nueva cruzada contra el comunismo ateo, en la que se sintieron tan cómodos Allen Dulles, Henry Luce, Reinhold Niebuhr, Sidney Hook o Irving Kristol. Era la línea para fomentar un hombre de fe que defendiese la civilización occidental.

McCarthy no fue la única que denunció esta nueva derecha; Fromm también lo hizo, más pausadamente, en una de las conferencias radiofónicas compiladas por Hans Jürgen Schultz cuando Fromm rondaba los ochenta años, el final de su vida: "Luego me afilié al Partido Socialista norteamericano, hasta que según mi opinión se había corrido tanto a la derecha que ni siquiera con el mayor de los optimismos era ya posible permanecer en él" (Fromm 1994: 185). Fromm sabía de lo que hablaba puesto que el había construido el armazón teórico de uno de los programas políticos del partido socialista (los veintinueve puntos de su Credo); él inició en 1955 la campaña "Ciudadanos en pro de la razón" -cuyo tercer borrador recogió las firmas de "Norman Thomas [...] Dan Bell [...] Lewis Mumford [...] y las sugerencias de Reinhold Niebuhr"- (Fromm 1993: 222 ss) y él fue posteriormente uno de los asesores para la alternativa humanista de la 
campaña a la carrera presidencial del Partido Demócrata de Eugene McCarthy en 1968; para finalmente afirmar, "al lado del autoritarismo fascista o estalinista y del supercapitalismo del tipo de la administración de los incentivos, la tercera de las grandes reacciones a la crítica del capitalismo es la teoría socialista [...] el socialismo democrático humanista" (Fromm 1986: 205 ss).

Así, durante la campaña de 1968, mientras Fromm asesoraba a uno de los candidatos demócratas, Donald Rumsfeld trabajaba para el candidato republicano Richard Nixon. Y aquí comenzó el engranaje de este nuevo grupo: Rumsfeld atrajo a Dick Cheney, Allan Bloom (discípulo de Leo Strauss) protegió a Paul Wolfowitz, que fue a la Universidad de Chicago para recibir clases también de Leo Strauss, y con ese legado Wolfowitz formó más tarde a Scooter Libby y a Francis Fukuyama. El filósofo Leo Strauss, judío de origen alemán refugiado en Estados Unidos, se convirtió por derecho propio en uno de los grandes teóricos del nuevo movimiento conservador que se estaba desarrollando. Sin embargo, en la campaña presidencial de 1972, para Kissinger la amenaza no era la derecha republicana ni los neo-conservadores demócratas del senador Jackson, sino la izquierda política contra la guerra de Vietnam y la campaña del demócrata McGovern. Craso error de apreciación de Kissinger: esa nueva derecha se hacía por momentos más fuerte en el interior del Partido Republicano, como bien conocía Rumsfeld.

Rumsfeld y Cheney, aprovechando la presencia en Washington para presidir la cena de la AFL$\mathrm{ClO}$ del Premio Nobel y exiliado soviético Aleksandr Solzhenitsyn, intentaron que el Presidente Ford se reuniese con él en la Casa Blanca. Este intento, aunque fue finalmente frustrado, constituyó el primer ataque a Kissinger y el primer paso para una de las mayores reestructuraciones ministeriales de las que ha sido objeto un gabinete ministerial estadounidense en su historia (la Masacre de Halloween). Dos senadores fueron gratificados: Rumsfeld, que sustituyó a Schlesinger como Secretario de Defensa, y Cheney, que se convirtió en el nuevo jefe de gabinete de la Casa Blanca. El nuevo director de la CIA fue George H. W. Bush (Mann 2007).

En este panorama, el antiguo representante principal de la sección de la Cruzada por la Libertad, Reagan, que elogiaba a Solzhenitsyn, lanzó su ataque frontal. Y los neoconservadores anticomunistas que se habían originado en el Partido Demócrata, como Jeane Kirkpatrick, criticaron duramente -en un artículo publicado en Commentary- al Presidente Carter por sus equivocaciones en el orden internacional al presionar tanto a diversos líderes para que se incrementasen, en demasía, las reformas democráticas. Artículo que Reagan premió de tal manera que terminó con el paso final de los neoconservadores al Partido Republicano -a lo que el propio Jackson no se opuso, precisamente- y con el que obtuvo su victoria en 1980, gracias al apoyo "inesperado" de un gran número de votantes, tradicionalmente demócratas.

Comenzó, de esta manera, el primer acto para el nuevo papel de Estados Unidos en el mundo, el concepto de Pax Americana por el que tanto habían luchado aquellos intelectuales ex-marxistas desde los inicios de la guerra fría en el marco incomparable de la Guerra Fría Cultural patrocinada por la CIA (Stonor Saunders 2001). 


\section{CONCLUSIONES}

Durante décadas la Internacional Comunista, Komintern, dirigió sutil y perfectamente a los intelectuales, artistas y científicos en sus campañas políticas internacionales por la Paz. Es más, tras la Segunda Guerra Mundial, se propuso hacerlo de nuevo para encubrir la invasión soviética de la Europa del Este. Fue la señal definitiva para que la Guerra fría se convirtiera también en el escenario de una intensísima batalla ideológica para ganarse las mentes y los corazones de los ciudadanos que deseaban luchar contra el totalitarismo soviético. Para este fin, la CIA y el IRD británico, con la ayuda de un grupo formado por antiguos propagandistas marxistas, fundarán una organización antiestalinista que no tardaría en atraer a gran parte de la intelectualidad mundial, desde la cual se forjarían las actividades del Congreso por la Libertad Cultural en el marco de la Guerra Psicológica para la Guerra Fría Cultural. En este entorno fue donde se engendró el proyecto ideológico de la izquierda no comunista, por el cual se apuntaló a la socialdemocracia europea en el bando de la democracia y el Estado del Bienestar.

Pero, a la postre, también cimentaron el desarrollo de un nuevo movimiento político que se fue gestando desde los sectores más duros del Congreso por la Libertad Cultural. El neoconservadurismo se conformó con unos intelectuales que peregrinaron desde el comunismo, pasando por el ala izquierdista del Partido Demócrata, hasta su integración en el Partido Republicano, donde llevaron a Reagan hasta la presidencia de Estados Unidos.

\section{BIBLIOGRAFÍA}

- $\quad$ Aron, Raymond, (1959). El mundo libre en la guerra fría. Valencia, Fomento de Cultura.

- Bell, Daniel, (1992). El fin de las ideologías. Madrid, Ministerio de Trabajo y Seguridad Social.

- $\quad$ Burham, James, (1943). La revolución de los directores. Buenos Aires, Claridad.

- $\quad$ Cavin, Susan, (2004). O.S.S. \& the Frankfurt School: Recycling the damaged lives of cultural outsiders', en http://convention3.allacademic.com/meta/p_mla_apa_research_citation/ 1/1/0/1/8/p110188_index.html

- $\quad$ Elola, Joseba, (2008). "Tres checos, un espía y un soplo", El País (Madrid), 26 octubre: 33 ss.

- $\quad$ Fromm, Erich, (1986). Psicoanálisis de la sociedad contemporánea. México D.F., Fondo de cultura económica.

- ـ _ 1994. El amor a la vida. Barcelona, Paidós Studio. 
- — _ 1993. Ética y política. Barcelona, Paidós.

- Ganser, Daniele, (2010). Los ejércitos secretos de la OTAN. La Operación Gladio y el terrorismo en Europa Occidental. Madrid, El Viejo Topo.

- García Guitián, Elena, (2001). El pensamiento político de Isaiah Berlin. Madrid, Centro de Estudios Políticos y Constitucionales.

- Glondys, Olga, (2007). Reivindicación de la Independencia Intelectual en la primera época de Cuadernos por la Libertad de la Cultura: I (marzo-mayo de 1953)-XXVII (noviembrediciembre de 1957). Trabajo de investigación del Departamento de Filología Española. Universidad Autónoma de Barcelona (UAB), en http://www.recercat.net/bitstream/2072/ $\underline{\text { 4359/1/Treball\%2Bde\%Brecercapdf }}$

- Judt, Tony, (2008). Sobre el olvidado SIGLO XX. Madrid, Taurus.

- 2010 Postguerra. Una historia de Europa desde 1945. Madrid, Taurus.

- Koestler, Arthur, (1951). El fracaso de un ídolo. Buenos Aires, Unión de editores latinos.

- 2008. "Kundera delató en 1950 a un espía checo", La Razón (Madrid), 14 octubre: 52 .

- Mann, James, (2007). Los Vulcanos. El gabinete de guerra de Bush. Granada, AlmED.

- $\quad$ Pardeiro, Marcos, (2008). “Represaliados en el olvido", La Razón (Madrid), 6 octubre: 24.

- Schlesinger, Arthur M., (1972). La política de la libertad. El centro vital. Barcelona, Dopesa.

- Stonor Saunders, Frances, (2001). La CIA y la guerra fría cultural. Madrid, Debate.

- $\quad$ Strauss, Leo, (2004). ¿Progreso o retorno? Barcelona, Paidós.

- _ _ _ 2007 Liberalismo antiguo y moderno. Buenos Aires, Katz Editores.

- $\quad$ VV.AA., (1975). Conversaciones sobre la nueva cultura. Barcelona, Kairós.

- VV.AA., (1973). Ensayos sobre el Apocalipsis. Barcelona, Kairós.

- Weiner, Tim, (2008). Legado de cenizas. La historia de la CIA. Barcelona, Debate.

- Winock, Michel, (2010). El siglo de los intelectuales. Barcelona, Edhasa. 